\title{
Dialectical Logic K-Model: A Mathematical Model for Machine
}

\author{
Yaozhi Jiang ${ }^{1}$ \\ ${ }^{1}$ Shijiazhuang High-tech district, Hebei, China \\ Correspondence: Yaozhi Jiang, Shijiazhuang High-tech district, Hebei, China. E-mail: jiangyaozhi@126.com
}

Received: September 11, 2017 Accepted: October 8, 2017 Online Published: October 27, 2017

doi:10.5539/jmr.v9n6p82

URL: https://doi.org/10.5539/jmr.v9n6p82

\begin{abstract}
An axiom system for dialectical logic K-model which based on Kirchhoff energy-method is established by author in the paper. The author describes that subjective-laws is the mirror imagine reflected from objective-laws and defines that the three-step which named by sensation, abstraction and thinking in artificial intelligence. At same time, describes that axiom system for dialectical logic K-model, in which contains such as logic-variable energy conservation law, Mozi's principle( mini-max principle) and forbidden law, etc. In the axiom system also contain such as a continuous true-value-function system valued on interval $[-1,+1]$ and the K-graph for logic-variable. And describes the giving value method by matrix based on $\mathrm{K}$-graph $\mathrm{G}_{\mathrm{K}}$ satisfied Kirchhoff laws to the logic variable. The author describes simply the linear and nonlinear logic variable system. And describes simply the logic variable involved three-dimension Euclidean space and topology networks space separately. Dialectical logic K-model would supply an computation algorithm idea for machine so that the machine is able to think by dialectical logic method, thus an important information-treated method maybe the dialectical logic.
\end{abstract}

Keywords: Artificial intelligence, Dialectical logic K-model, K-graph $\mathrm{G}_{\mathrm{K}}$, Kirchhoff-laws, Contradiction-function matrix

AMS(MSC2010): 03B99

\section{Introduction}

Objective world is consist of five factors which are MATTER, ENERGY, INFORMATION, SPACE and TIME. Among these factors, there are many inner or outer laws of causation can be called as objective-laws, also can be called as objective-logic. Corresponding to objective-laws, there is a mirror image within machine of artificial intelligence, which can be called as subjective-laws, also can be called as subjective-logic. The machine of artificial intelligence only holds objective-laws through subjective-laws, or with another word, only holds objective-logic through subjective-logic. Subjective-logic is built on infinite times circulation by the three-step which named Sensation, Abstraction and Thinking. Sensation is input-ability to machine from objective information with sensors. Abstraction is conception -classification-ability to sense information and to name it. Thinking is ability of machine researching from conceptionclassification information to get the subjective-logic.

The dialectical logic was established by Hegel, a great philosopher (Bencivenga, E., 2000; Kosok, M., 1966). By the opinion of dialectical logic, the objective laws are dynamical, varied and driven by the causality law. Its main opinion is: contradictions are the causes of object's variety. The establishment of mathematical model for dialectical logic is the necessary condition in order to make that the dialectical logic is compute-able in machine.

Dialectical logic K-model established in the paper would supply a computation-idea for machine so that the machine is able to think by dialectical logic method, so an important information-treated method would be the dialectical logic. The dialectical logic K-model combined with three main recognition technologies (image recognition technology, voice recognition technology, text recognition technology), data base technology and machine-self-programming technology will take a great progress to artificial intelligence.

\section{Axiom System and Theorems}

\subsection{Conception and the Conception-dimension}

2.1.1. The Conception is the Name Which be Named the Object-thing's Main Property and neglected its secondary properties. Conception is the basis of artificial intelligence.

\subsubsection{The Conception-dimension}

The conception-dimension is the dimension-number of conception. For example, HORSE is an one-dimension 
conception; WHITE HORSE is a two-dimension conception; RUNNING WHITE HORSE is a three-dimension conception; ONE RUNNING WHITE HORSE is a four-dimension conception; etc.

\subsubsection{Associate Database}

The three main recognition technologies will setup three data bases, ( although there are other data base, for example physical data base and chemical data base, etc. ) These three data bases are associated by the conception-dimension.

\subsection{Several Laws}

\subsubsection{Definitions}

Denote objective-domain by B, and subjective-domain by A; define research operator $\nabla$ (research operator is an arithmetic to get subjective-logic from objective-logic) and inverse research operator $\nabla^{-1}$ (inverse research operator is an arithmetic which make subjective-logic to objective-logic in order to check its true or false) and thus

$$
\begin{aligned}
\nabla\left(B_{\mathrm{i}} \in \mathrm{B}\right) \Leftrightarrow \nabla\left(A_{\mathrm{i}} \in \mathrm{A}\right) \\
\nabla^{-1} \nabla\left(B_{\mathrm{i}} \in \mathrm{B}\right) \Leftrightarrow \nabla^{-1} \nabla\left(A_{\mathrm{i}} \in \mathrm{A}\right) \\
\cdots \\
\nabla^{-1} \nabla \cdots \nabla^{-1} \nabla\left(B_{\mathrm{i}} \in \mathrm{B}\right) \Leftrightarrow \nabla^{-1} \nabla \cdots \nabla^{-1} \nabla\left(A_{\mathrm{i}} \in \mathrm{A}\right) \\
\nabla^{-1} \nabla=F_{1} \\
\quad \cdots \\
\underbrace{\nabla^{-1} \nabla \cdots \nabla^{-1} \nabla \nabla^{-1} \nabla}_{\mathrm{j} \text { couplesof } \nabla^{-1} \nabla}=F_{\mathrm{j}}
\end{aligned}
$$

then

$$
F_{\mathrm{j}}\left(B_{\mathrm{i}} \in \mathrm{B}\right) \Leftrightarrow F_{\mathrm{j}}\left(A_{\mathrm{i}} \in \mathrm{A}\right)
$$

As above, $F_{j}$ is the $j$-th cycle research operator, obviously $F_{j}$ is a logic-transformation between domain A and domain B. Sometimes can denote $F_{j}$ by $F$. Symbol $\Leftrightarrow$ is denoted that the object logic and the subject logic are mirror imagine each other.

Denote subjective-logic variable by

$$
A_{i}^{ \pm n}(t)=\left\{a_{1}^{ \pm}(t), a_{2}^{ \pm}(t), \cdots, a_{n}^{ \pm}\right\}
$$

as above, $t$ is time variable, right superscript $\pm n$ denote the logic variable contains $n$ couples of contradicting sub variable $a_{i}^{ \pm}(t), n$ is called the rank-number of $A_{i}^{ \pm n}(t)$, right subscript $i$ denotes the $i$ - th causation law.

$T\left(A_{i}^{ \pm n}(t)\right)$ is denoted the true-value function of logic variable $A_{i}^{ \pm n}(t)$, and $T\left(A_{i}^{ \pm n}(t)\right) \in[-1,+1]$.

Denote the mirror image in objective-domain of $A_{i}^{ \pm n}(t) \in \mathrm{A}$ by $B_{i}(t) \in \mathrm{B}$.

\subsubsection{Objective Research-Able Law}

To every objective-logic variable $B_{i}(t) \in \mathrm{B}$ there must always exist logic-transformation $F$, make

$$
A_{i}^{ \pm n}(t)=F\left(B_{i}(t)\right)
$$

\subsubsection{Research Error Alternating-convergence Law}

To every correct logic-transformation $F$, always make

$$
\lim _{j \rightarrow \infty} \mathrm{C}\left|A_{i}^{ \pm n}(t)-B_{i}(t)\right|=\lim _{j \rightarrow \infty} \mathrm{C}\left|F_{j}\left(B_{i}(t)\right)-B_{i}(t)\right|=0
$$

i.e. to every finite $j<\infty$, error-function

$$
E_{j}(t)=\left|F_{j}\left(B_{i}(t)\right)-B_{i}(t)\right|>0
$$


in the formulation as above $\mathrm{C}=+1$, if $\mathrm{j}=2 \mathrm{p}+1$; or $\mathrm{C}=-1$, if $\mathrm{j}=2 \mathrm{p}, \mathrm{p}=0,1,2, \cdots$

In fact, incorrect logic-transformation $F^{*}$ can bring the researching divergence. The faster the convergence of the operator $F_{j}$ is, the brighter the operator $F_{j}$ is.

2.2.4. Logic Variable Energy Conservation Law

For logic variable $A_{i}^{ \pm n}(t)$, its inner producing-energy $E_{i}$ is equal to the consumed work $J_{i}$ by its

contradiction-function(see the 10.4 contradiction-function as below), i.e.

$$
E_{i}=J_{i}
$$

2.2.5.Mozi’s Principle (Mini-Max Principle)(Johnston, Ian, 2010)

Logic variable $A_{i}^{ \pm n}(t)$ in changing, must be satisfied or must be satisfied asymptotically by that cost-function $C\left(A_{i}^{ \pm n}(t)\right)$ is minimum and gain-function $\mathrm{G} G\left(A_{i}^{ \pm n}(t)\right)$ is maximum. Denote the pure gain-function by $L\left(A_{i}^{ \pm n}(t)\right)$, i.e.

$$
L\left(A_{i}^{\mp n}(t)\right)=\max \left(G\left(A_{i}^{ \pm n}(t)\right)-\min C\left(A_{i}^{ \pm n}(t)\right)\right)
$$

or

$$
\lim _{t \rightarrow \infty} L\left(A_{i}^{ \pm n}(t)\right)=\max _{t \rightarrow \infty}\left(G\left(A_{i}^{ \pm n}(t)\right)\right)-\min _{t \rightarrow \infty} C\left(A_{i}^{ \pm n}(t)\right)
$$

\subsubsection{Memory-inertia Law}

\subsubsection{Last-time Memory Law}

For time sequence $t_{1}<t_{2}<\cdots<t_{k}<\cdots$, denote the memory effect-weight-function of contradiction sub variable $a_{i}^{ \pm}\left(t_{p}\right)$ from time $t$ by $R\left(a_{i}^{ \pm}\left(t_{p}\right)\right)$

$$
R\left(a_{i}^{ \pm}\left(t_{p}\right)\right) \in[0,1]
$$

and

$$
R\left(a_{i}^{ \pm}\left(t_{k}\right)\right)>R\left(a_{i}^{ \pm}\left(t_{j}\right)\right)
$$

If and only if $t_{k}>t_{j}$, then memory prefer the $a_{i}^{ \pm}\left(t_{k}\right)$ of that one by the last time.

\subsubsection{Importance Memory Law}

For importance sequence $M\left(a_{p}^{ \pm}(t)\right)<M\left(a_{q}^{ \pm}(t)\right)<\cdots<M\left(a_{r}^{ \pm}(t)\right)$, and $M\left(a_{i}^{ \pm}(t)\right) \in[0,1]$

So the memory prefer the $a_{r}^{ \pm}(t)$, if and only if its $M\left(a_{r}^{ \pm}(t)\right)$ is bigger one.

2.2.6.3. Bigger Probability Memory Law

For property event $\alpha_{1}, \alpha_{2}, \cdots, \alpha_{k}$, their corresponding probability are

$$
P\left(\alpha_{1}\right), P\left(\alpha_{2}\right), \cdots, P\left(\alpha_{k}\right),
$$

and

$$
p\left(\alpha_{n}\right)>p\left(\alpha_{m}\right)
$$

so the memory prefer $\alpha_{n}$.

2.3 Machine Self-programmable and Self-correctable Law

In the researching process, the machine must have ability to self-programmable and self-correctable without the operations by human beings.

\subsection{Forbidden Law}

There are two kinds of logic: one of them is dialectical-logic corresponding to intelligence quotient and another of them is 
imagine-logic corresponding to emotional quotient.

The imagine-logic would be forbidden to put into machine, because the imagine-logic will make machine to produce emotional quotient so that the autonomous-mind will belong to the machine. The artificial intelligence with the autonomous-mind will not like to be "a willing tool" for human beings, so "a new creation" will be created, of cause this is harmful to human beings.

\subsection{Logic Variable Infinite-separable-characteristic Law}

The logic variable is infinite-separable, i.e.

$$
A_{i}^{ \pm n}(t)=\lim _{n \rightarrow \infty}\left\{a_{1}^{ \pm}(t), a_{2}^{ \pm}(t), \cdots, a_{n}^{ \pm}(t)\right\}
$$

\subsection{Logical Inductive and Deductive Method Theorem}

For cycle research operator arithmetic $F_{j}$, make

$$
A_{i}^{ \pm n}(t)=F_{j}\left(B_{i}(t)\right)
$$

2.6.1. (inductive) if as above is true to finite $F_{k}, k=1,2, \cdots, j$

2.6.2. (hypothesis) suppose as above is true to $F_{k+1}$,

2.6.3. (deductive) so that as above is true to all $j<\infty$.

Proof: through finite to prove infinite, there always exists error-function based on the 2.2.3. research error alternating-convergence law, if the 2.6.2. (hypothesis) is false, then producing the false to the 2.6.3. (deductive).Thus the truth to every step of $F_{j}$ would be carefully checked.

Proof is over.

\subsection{Definitions of Logic Algorithm(Boolean operator)}

For $A_{i}^{ \pm r}(t), A_{i}^{ \pm s}(t), A_{i}^{ \pm k}(t)$, denote their true value function by corresponding to $T\left(A_{i}^{ \pm r}(t)\right), T\left(A_{i}^{ \pm s}(t)\right)$,

$T\left(A_{i}^{ \pm k}(t)\right)$

\subsubsection{Logic $U$ (Boolean OR)}

Definition $T\left(A_{i}^{ \pm s}(t)\right) \bigcup T\left(A_{i}^{ \pm r}(t)\right)=\max \left\{T\left(A_{i}^{ \pm s}(t)\right) ; T\left(A_{i}^{ \pm r}(t)\right)\right\}$

logic $U$ is satisfied by as below

commutative law $T\left(A_{i}^{ \pm s}(t)\right) \bigcup T\left(A_{i}^{ \pm r}(t)\right)=T\left(A_{i}^{ \pm r}(t)\right) \bigcup T\left(A_{i}^{ \pm s}(t)\right)$

associative law $\left(T\left(A_{i}^{ \pm s}(t)\right) \bigcup T\left(A_{i}^{ \pm r}(t)\right)\right) \bigcup T\left(A_{i}^{ \pm k}(t)\right)=T\left(A_{i}^{ \pm s}(t)\right) \bigcup\left(T\left(A_{i}^{ \pm r}(t)\right) \bigcup T\left(A_{i}^{ \pm k}(t)\right)\right)$

\subsubsection{Logic $\bigcap$ (Boolean AND)}

definition $T\left(A_{i}^{ \pm s}(t)\right) \bigcap T\left(A_{i}^{ \pm r}(t)\right)=\min \left\{T\left(A_{i}^{ \pm s}(t)\right) ; T\left(A_{i}^{ \pm r}(t)\right)\right\}$

logic $\bigcap$ is satisfied by as below

commutative law $T\left(A_{i}^{ \pm s}(t)\right) \bigcap T\left(A_{i}^{ \pm r}(t)\right)=T\left(A_{i}^{ \pm r}(t)\right) \bigcap T\left(A_{i}^{ \pm s}(t)\right)$

associative law $\left(T\left(A_{i}^{ \pm s}(t)\right) \bigcap T\left(A_{i}^{ \pm r}(t)\right)\right) \bigcap T\left(A_{i}^{ \pm k}(t)\right)=T\left(A_{i}^{ \pm s}(t)\right) \bigcap\left(T\left(A_{i}^{ \pm r}(t)\right) \bigcap T\left(A_{i}^{ \pm k}(t)\right)\right)$

\subsubsection{Logic Hybrid Arithmetic (Boolean OR \& Boolean AND)}

Logic hybrid arithmetic is satisfied as below 
distributive law

$$
T\left(A_{i}^{ \pm s}(t)\right) \bigcup\left(T\left(A_{i}^{ \pm r}(t)\right) \bigcap T\left(A_{i}^{ \pm k}(t)\right)\right)=\left(T\left(A_{i}^{ \pm s}(t)\right) \bigcup T\left(A_{i}^{ \pm r}(t)\right)\right) \bigcap\left(T\left(A_{i}^{ \pm s}(t)\right) \bigcup T\left(A_{i}^{ \pm k}(t)\right)\right)
$$

\subsubsection{Logic $N$ (Boolean NOT)}

$N\left[T\left(A_{i}^{ \pm n}(t)\right)\right]= \pm 1-T\left(A_{i}^{ \pm n}(t)\right)$, if $T\left(A_{i}^{ \pm n}(t)\right)>0$ is positive in front of the 1 ; if $T\left(A_{i}^{ \pm n}(t)\right)<0$ is negative in front of the 1 .

Denote $\underbrace{N\left[N\left[\cdots N\left[A_{i}^{ \pm n}(t)\right] \cdots\right]\right]}_{p}=N^{p}\left[A_{i}^{ \pm n}(t)\right]$

logic $N$ is satisfied by as below

idempotent law $N^{p}\left[A_{i}^{ \pm n}(t)\right]=A_{i}^{ \pm n}(t)$, if $p=2 k$;

$$
N^{p}\left[A_{i}^{ \pm n}(t)\right]=N\left[A_{i}^{ \pm n}(t)\right], p=2 k+1, k=1,2, \cdots, m, \cdots
$$

2.8 De Morgan's Theorem

$$
\begin{aligned}
& N\left[T\left(A_{i}^{ \pm s}(t)\right) \bigcap T\left(A_{i}^{\mp r}(t)\right)\right]=N\left[T\left(A_{i}^{ \pm s}(t)\right)\right] \bigcup N\left[T\left(A_{i}^{ \pm r}(t)\right)\right] \\
& N\left[T\left(A_{i}^{ \pm s}(t)\right) \bigcup T\left(A_{i}^{ \pm r}(t)\right)\right]=N\left[T\left(A_{i}^{\mp s}(t)\right)\right] \bigcap N\left[T\left(A_{i}^{ \pm r}(t)\right)\right]
\end{aligned}
$$

Proof:

$$
\begin{aligned}
& N\left[T\left(A_{i}^{ \pm s}(t)\right) \bigcap T\left(A_{i}^{ \pm r}(t)\right)\right]= \pm 1-\left(T\left(A_{i}^{ \pm s}(t)\right) \bigcap T\left(A_{i}^{ \pm r}(t)\right)\right)= \\
& = \pm 1-\min \left\{T\left(A_{i}^{ \pm s}(t)\right) ; T\left(A_{i}^{ \pm r}(t)\right)\right\}=\max \left\{ \pm 1-T\left(A_{i}^{ \pm s}(t)\right) ; \pm-T\left(A_{i}^{ \pm r}(t)\right)\right\}= \\
& =N\left[T\left(A_{i}^{ \pm s}(t)\right)\right] \bigcup N\left[T\left(A_{i}^{ \pm r}(t)\right)\right]
\end{aligned}
$$

The proof of formulation(1) is over.

$$
\begin{aligned}
& N\left[T\left(A_{i}^{ \pm s}(t)\right) \bigcup T\left(A_{i}^{ \pm r}(t)\right)\right]= \pm 1-\left(T\left(A_{i}^{ \pm s}(t)\right) \bigcup T\left(A_{i}^{ \pm r}(t)\right)\right)= \\
& = \pm 1-\max \left\{T\left(A_{i}^{ \pm s}(t)\right) ; T\left(A_{i}^{\mp r}(t)\right)\right\}=\min \left\{ \pm 1-T\left(A_{i}^{ \pm s}(t)\right) ; \pm 1-T\left(A_{i}^{ \pm r}(t)\right)\right\}= \\
& =\min \left\{N\left[T\left(A_{i}^{ \pm s}(t)\right)\right] ; T\left(A_{i}^{ \pm r}(t)\right)\right\}= \\
& =N\left[T\left(A_{i}^{ \pm s}(t)\right)\right] \cap N\left[T\left(A_{i}^{ \pm r}(t)\right)\right]
\end{aligned}
$$

The proof of formulation(2) is over.

Remark1: As shown above, when time $t$ is degenerated into a constant and destroy the contradictions in the ormulations, then the formulation (1) and (2) will be degenerated from the continuous true value function Boolean algebra valued on interval $[-1,+1]$ into the mathematical model of formal logic, i.e. binary Boolean algebra.

\subsection{Logic True Valued Function Composition Theorem}

For logic true valued function $T\left(A_{i}^{ \pm s}(t)\right), T\left(A_{i}^{ \pm r}(t)\right)$, as below there is a composition theorem:

If equation $T\left(A_{i}^{ \pm s}(t)\right)-T\left(A_{i}^{ \pm r}(t)\right)=0$, exist a root-set

$$
T=\left\{t_{1}, t_{2}, \cdots, t_{m}\right\}
$$

and 


$$
t_{1}<t_{2}<\cdots<t_{m}
$$

so that

$$
\begin{aligned}
& \max \left\{T\left(A_{i}^{ \pm s}(t)\right) ; T\left(A_{i}^{ \pm r}(t)\right)\right\}=\max _{t \in\left[t_{0}, t_{1}\right]}\left\{T\left(A_{i}^{ \pm s}(t)\right) ; T\left(A_{i}^{ \pm r}(t)\right)\right\}+\max _{t \in\left[t_{1}, t_{2}\right]}\left\{T\left(A_{i}^{ \pm s}(t)\right) ; T\left(A_{i}^{ \pm r}(t)\right)\right\}+\cdots+ \\
& +\max _{t \in\left[t_{m} ; t\right]}\left\{T\left(A_{i}^{ \pm s}(t)\right) ; T\left(A_{i}^{ \pm r}(t)\right)\right\}
\end{aligned}
$$

as similar as above, also

$$
\begin{aligned}
& \min \left\{T\left(A_{i}^{ \pm s}(t)\right) ; T\left(A_{i}^{ \pm r}(t)\right)\right\}=\min _{t \in\left[t_{0}, t_{1}\right]}\left\{T\left(A_{i}^{ \pm s}(t)\right) ; T\left(A_{i}^{ \pm r}(t)\right)\right\}+\min _{t \in\left[t_{1}, t_{2}\right]}\left\{T\left(A_{i}^{ \pm s}(t)\right) ; T\left(A_{i}^{ \pm r}(t)\right)\right\}+\cdots+ \\
& +\min _{t \in\left[t_{m}, t\right]}\left\{T\left(A_{i}^{ \pm s}(t)\right) ; T\left(A_{i}^{ \pm r}(t)\right)\right\}
\end{aligned}
$$

If equation $T\left(A_{i}^{ \pm s}(t)\right)-T\left(A_{i}^{ \pm r}(t)\right)=0$ in which the possible augment root and complex root will be removed out.

If $T\left(A_{i}^{ \pm s}(t)\right)-T\left(A_{i}^{ \pm r}(t)\right) \neq 0$, so the root-set $T=\phi$ that $\max \left\{T\left(A_{i}^{ \pm s}(t)\right) ; T\left(A_{i}^{ \pm r}(t)\right)\right\}$ or

$\min \left\{T\left(A_{i}^{ \pm s}(t)\right) ; T\left(A_{i}^{ \pm r}(t)\right)\right\}$ in which select a maximum or minimum of pure function.

Proof:obviously based on definition of logic arithmetic.

Proof is over.

2.10 Linear Logical System And Non-Linear Logical System

According to the 2.2.1. as shown above, obviously we have the formulation as below

$$
F_{i}\left(A_{s}^{ \pm n}(t)\right)=T_{i}\left(A_{s}^{ \pm n}(t)\right)
$$

\subsubsection{Definition}

Logic variable $A_{s}^{ \pm n}(t)$ and $A_{r}^{ \pm n}(t)$ are named linear logical system, if and only if they are satisfied by

2.10.1.1,2.10.1.2 and 2.10.1.3 as below; otherwise can be named non-linear logical system:

2.10.1.1. $F_{i}\left(A_{s}^{ \pm n}(t) \bigcup A_{r}^{ \pm n}(t)\right)=F_{i}\left(A_{s}^{ \pm n}(t)\right) \bigcup F_{i}\left(A_{r}^{ \pm n}(t)\right)=T_{i}\left(A_{s}^{ \pm n}(t)\right) \cup T_{i}\left(A_{r}^{ \pm n}(t)\right)$

2.10.1.2. $F_{i}\left(A_{s}^{ \pm n}(t) \bigcap A_{r}^{ \pm n}(t)\right)=F_{i}\left(A_{s}^{\mp n}(t)\right) \bigcap F_{i}\left(A_{r}^{ \pm n}(t)\right)=T_{i}\left(A_{s}^{ \pm n}(t)\right) \bigcap T_{i}\left(A_{r}^{ \pm n}(t)\right)$

2.10.1.3. $F_{i}\left(N\left(A_{s}^{ \pm n}(t)\right)\right)=N\left(F_{i}\left(A_{s}^{ \pm n}(t)\right)\right)=N\left(T_{i}\left(A_{s}^{ \pm n}(t)\right)\right)$

Remark 2: The strong action influence by human beings to the object would produce non-linear logic; the weaker action influence or zero action influence by human beings to the object would produce linear logic. Because of, the error action influence of human beings would produce the non-linear properties. An example for linear logic is science, and an example for non-linear logic is engineering or technology. As similar as above , another example for linear logic is original ecology system, and another example for non-linear logic is a planted system in agriculture .

2.11 Expression by graph $\mathrm{G}_{\mathrm{K}}$ to logic variable $A_{i}^{ \pm n}(t)$ (Berge, C., 1973)

2.11.1. Kirchhoff Power-Function Law And Kirchhoff Flow-Function Law

2.11.1.1 Defines a connecting directed simple graph $\mathrm{G}_{\mathrm{K}}$ with $2(\mathrm{n}+1)$ nodes and $\mathrm{E}$ edges $\mathrm{G}_{\mathrm{K}}=\left\{\mathrm{V}_{2(\mathrm{n}+1)} ; \mathrm{E}\right\}$ 
In graph $\mathrm{G}_{\mathrm{K}}$ every node will be given a power-function $\mathrm{v}_{\mathrm{i}}(t)$ for node $\mathrm{V}_{\mathrm{i}}$ and node $\mathrm{V}_{\mathrm{j}}$, if edge, $\mathrm{E}_{\mathrm{ij}} \in \mathrm{E}$, then on the edge $E_{i j}$ exists a power-function

$$
\mathrm{e}_{\mathrm{ij}}(t)=\mathrm{v}_{\mathrm{j}}(t)-\mathrm{v}_{\mathrm{i}}(t)
$$

In formulation (3), lower reaches node is left and upper reaches node is right, in the opposite direction would sign a negative in the front of $\mathrm{e}_{\mathrm{ij}}(t)$.

In the graph $G_{K}$, every cycle $W_{i j}$ is satisfied by

$$
\sum_{\text {if }} \mathrm{E}_{\mathrm{ij}} \in \mathrm{e}_{\mathrm{ij}}(\mathrm{t})=0
$$

\subsubsection{Kirchhoff Flow-function Law}

In the graph $G_{K}$, every node $V_{i}$ is satisfied by

$$
\sum_{\text {if }} \mathrm{f}_{\mathrm{i}} \in \mathrm{V}_{\mathrm{i}}(t)=0
$$

as above input flow $\mathrm{f}_{\mathrm{i}}(t)$ is positive and output flow $\mathrm{f}_{\mathrm{i}}(t)$ is negative.

2.11.1.4. Contradiction -function

Defines

$$
\mathrm{R}_{\mathrm{ij}}(t)=\mathrm{e}_{\mathrm{ij}}(t) / \mathrm{f}_{\mathrm{ij}}(t)
$$

is the contradiction-function of edge $E_{i j}$ in graph $G_{K}$.

2.11.1.5. Work And Energy Law

In the graph $G_{K}$ every edge $E_{i j}$, make

$$
\mathrm{W}_{\mathrm{ij}}=\int_{t_{0}}^{t_{1}} \mathrm{e}_{\mathrm{ij}}(t) \mathrm{f}_{\mathrm{ij}}(t) d t
$$

$\mathrm{W}_{\mathrm{ij}}$ is called that work done in $\left[t_{0}, t_{1}\right]$ by contradiction-function $\mathrm{R}_{\mathrm{ij}}(t)$ on edge $\mathrm{E}_{\mathrm{ij}}$.

2.11.2. Structure in Graph $\mathrm{G}_{\mathrm{K}}$ of Logic-variable $A_{i}^{ \pm n}(t)$

2.11.2.1. Graph $\mathrm{G}_{\mathrm{K}}$ have $\mathrm{n}$ Couples of Nodes and Two Nodes $\mathrm{V}_{+1}, \mathrm{~V}_{-1}$, total $2(\mathrm{n}+1)$;

2.11.2.2. Graph $\mathrm{G}_{\mathrm{K}}$ is a no-loop, no-multiple edge directed simple graph;

2.11.2.3. In the graph $\mathrm{G}_{\mathrm{K}}, \mathrm{n}+1$ positive nodes construct a perfect sub graph, another $\mathrm{n}+1$ negative nodes construct another perfect sub graph; 
2.11.2.4. Node $\mathrm{V}_{+1}$ connect only to node $\mathrm{V}_{-1}$, positive node $\mathrm{V}_{\mathrm{q}}^{+}$connect only to negative node $\mathrm{V}_{\mathrm{q}}^{-}$which right subscript is equal.

2.11.2.5. The power-function of node $\mathrm{V}_{+1}$ is a constant +1 , the power-function of $\mathrm{V}_{-1}$ is a constant -1 , the power-function of edge $E_{+1,-1}$ is a constant +2 , the flow-function of edge $E_{+1,-1}$ is a constant I;

2.11.2.6. In the graph $\mathrm{G}_{\mathrm{K}}$, other nodes and edges all be defined power-function $\mathrm{v}_{\mathrm{i}}(t)$, power-function $\mathrm{e}_{\mathrm{ij}}(t)$,

flow-function $\mathrm{f}_{\mathrm{ij}}(t)$ and contradiction-function $\mathrm{R}_{\mathrm{ij}}(t)$ on them, these are all satisfied by Kirchhoff laws as above.

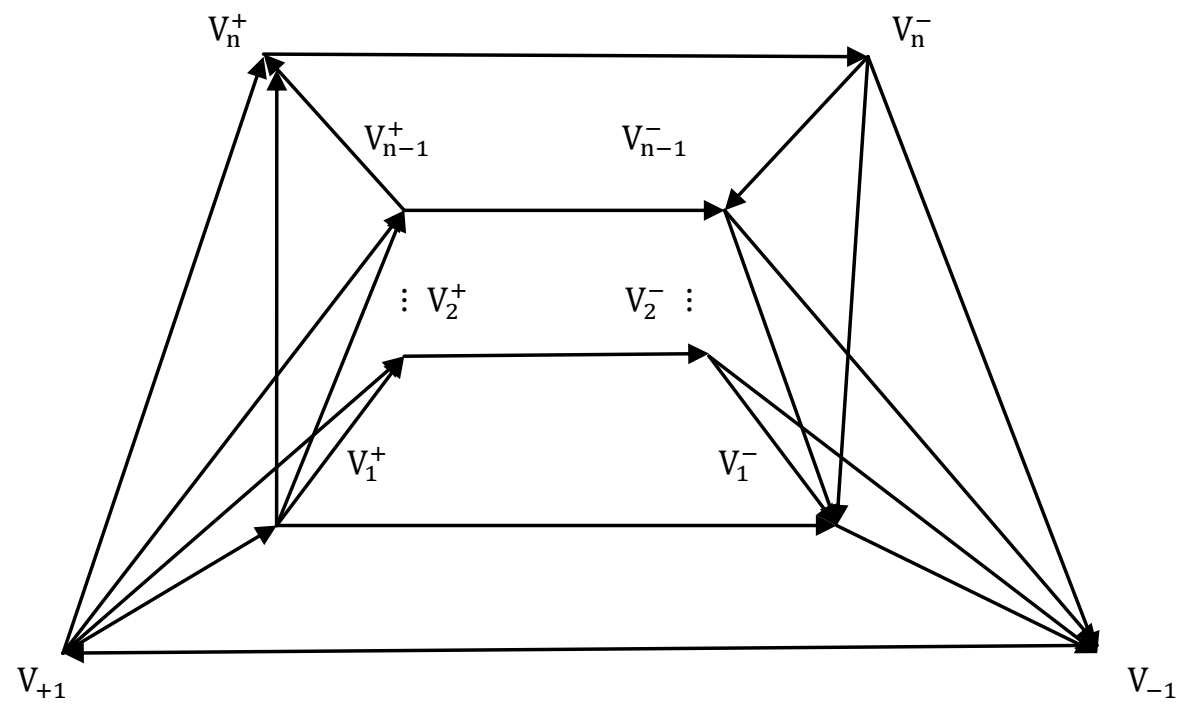

Fig.1 the structure of graph $\mathrm{G}_{\mathrm{K}}$

2.11.3. The giving value to logic variable $A_{i}^{ \pm n}(t)$ by graph $\mathrm{G}_{\mathrm{K}}$

Corresponding to every logic variable, there must exist a graph $G_{K}$ in order to give the true value to the logic variable $A_{i}^{ \pm n}(t)$. The true value is given by the formulation as below

$$
T\left(A_{i}^{ \pm n}(t)\right)=\frac{1}{2 \mathrm{n}} \sum_{\mathrm{i}=1}^{\mathrm{n}}\left(\mathrm{v}_{\mathrm{i}}^{+}(t)+\mathrm{v}_{\mathrm{i}}^{--}(t)\right)
$$

$T\left(A_{i}^{ \pm n}(t)\right) \in[-1,+1]$, if $T\left(A_{i}^{ \pm n}(t)\right)=0$ then the positive factor and negative factor which formed the two sides of the contradiction are balance; if $T\left(A_{i}^{ \pm n}(t)\right)>0$ then the positive factor in the contradiction is superior factor; if $T\left(A_{i}^{ \pm n}(t)\right)<0$ then the negative factor in the contradiction is superior factor. 
2.12 Expression by power function matrix $M_{K E}$, flow function matrix $M_{K F}$ and contradiction function matrix $M_{K C}$

for logic variable $A_{i}^{ \pm n}(t)$

The expression by matrix can make logic variable $A_{i}^{ \pm n}(t)$ is compute-able in machine.

2.12.1. Power function matrix $M_{K E}$

The elements of power function matrix $M_{K E}$ are:

$\mathrm{a}$, at the first row is the power function value $\mathrm{v}_{+1}(t), \mathrm{v}_{-1}(t), \mathrm{v}_{1}^{+}(t), \mathrm{v}_{2}^{+}(t), \cdots, \mathrm{v}_{\mathrm{n}}^{+}(t), \mathrm{v}_{1}^{-}(t), \mathrm{v}_{2}^{-}(t), \cdots, \mathrm{v}_{\mathrm{n}}^{-}(t)$ of the nodes $\mathrm{V}_{+1}, \mathrm{~V}_{-1}, \mathrm{~V}_{1}^{+}, \mathrm{V}_{2}^{+}, \cdots, \mathrm{V}_{\mathrm{n}}^{+}, \mathrm{V}_{1}^{-}, \mathrm{V}_{2}^{-}, \cdots, \mathrm{V}_{\mathrm{n}}^{-}$;

b. at the first column is the transposed vector of the first row ;

c. the other matrix elements are: $e_{i j}=e_{i j}(t)$, if edge $e_{i j}$ exist; otherwise $e_{i j}=0$. Thus the power function matrix is

$$
M_{K E}=\left|\begin{array}{ccccccccc} 
& +1 & -1 & \mathrm{v}_{1}^{+}(t) & \cdots & \mathrm{v}_{\mathrm{n}}^{+}(t) & \mathrm{v}_{1}^{-}(t) & \cdots & \mathrm{v}_{\mathrm{n}}^{-}(t) \\
+1 & 0 & +2 & \mathrm{v}_{1}^{+}(t)-1 & \cdots & \mathrm{v}_{\mathrm{n}}^{+}(t)-1 & 0 & \cdots & 0 \\
-1 & -2 & 0 & 0 & \cdots & 0 & -1-\mathrm{v}_{1}^{-}(t) & \cdots & -1-\mathrm{v}_{\mathrm{n}}^{-}(t) \\
\mathrm{v}_{1}^{+}(t) & \mathrm{v}_{1}^{+}(t)-1 & 0 & 0 & \cdots & \mathrm{v}_{\mathrm{n}}^{+}(t)-\mathrm{v}_{1}^{+}(t) & \mathrm{v}_{1}^{-}(t)-\mathrm{v}_{1}^{+}(t) & \cdots & 0 \\
\vdots & \vdots & \vdots & \vdots & \ddots & \vdots & \vdots & \vdots & \vdots \\
\mathrm{v}_{\mathrm{n}}^{+}(t) & 1-\mathrm{v}_{\mathrm{n}}^{+}(t) & 0 & \mathrm{v}_{1}^{+}(t)-\mathrm{v}_{\mathrm{n}}^{+}(t) & \cdots & 0 & 0 & \cdots & \mathrm{v}_{\mathrm{n}}^{-}(t)-\mathrm{v}_{\mathrm{n}}^{+}(t) \\
\mathrm{v}_{1}^{-}(t) & 0 & \mathrm{v}_{1}^{-}(t)+1 & \mathrm{v}_{1}^{+}(t)-\mathrm{v}_{1}^{-}(t) & \cdots & 0 & 0 & \cdots & \mathrm{v}_{1}^{-}(t)-\mathrm{v}_{\mathrm{n}}^{-}(t) \\
\vdots & \vdots & \vdots & \vdots & \vdots & \vdots & \vdots & \ddots & \vdots \\
\mathrm{v}_{\mathrm{n}}^{-}(t) & 0 & -1-\mathrm{v}_{\mathrm{n}}^{-}(t) & 0 & \cdots & \mathrm{v}_{\mathrm{n}}^{+}(t)-\mathrm{v}_{\mathrm{n}}^{-}(t) \mathrm{v}_{1}^{-}(t)-\mathrm{v}_{\mathrm{n}}^{-}(t) \cdots & 0
\end{array}\right|
$$

2.12.2. Flow function matrix $M_{K F}$

The matrix elements of the flow function matrix $M_{K F}$ are

a. at the first row is the node vector $\mathrm{V}_{+1}, \mathrm{~V}_{-1}, \mathrm{~V}_{1}^{+}, \mathrm{V}_{2}^{+}, \cdots, \mathrm{V}_{\mathrm{n}}^{+}, \mathrm{V}_{1}^{-}, \mathrm{V}_{2}^{-}, \cdots, \mathrm{V}_{\mathrm{n}}^{-}$;

b. at the first column is transposed vector of the first row;

c. The other elements of flow function matrix $M_{K F}$ are: $\mathrm{e}_{\mathrm{ij}}=\mathrm{f}_{\mathrm{ij}}(t)$, if the edge $\mathrm{e}_{\mathrm{ij}}$ exist;otherwise $\mathrm{e}_{\mathrm{ij}}=0$.

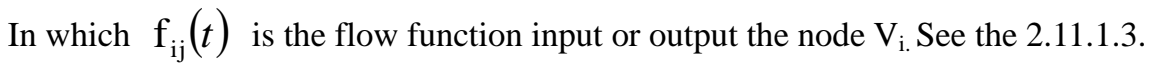

Then the flow function matrix 


$$
M_{K F}=\left|\begin{array}{ccccccccc} 
& \mathrm{V}_{+1} & \mathrm{~V}_{-1} & \mathrm{~V}_{1}^{+} & \cdots & \mathrm{V}_{\mathrm{n}}^{+} & \mathrm{V}_{1}^{-} & \cdots & \mathrm{V}_{\mathrm{n}}^{-} \\
\mathrm{V}_{+1} & 0 & \mathrm{I} & -\mathrm{f}_{+1,1^{+}}(t) & \cdots & -\mathrm{f}_{+1, \mathrm{n}^{+}}(t) & 0 & \cdots & 0 \\
\mathrm{~V}_{-1} & -\mathrm{I} & 0 & 0 & \cdots & 0 & \mathrm{f}_{-1,1^{-}}(t) & \cdots & \mathrm{f}_{-1, \mathrm{n}^{-}}(t) \\
\mathrm{V}_{1}^{+} & \mathrm{f}_{1^{+},+1}(t) & 0 & 0 & \cdots & -\mathrm{f}_{1^{+}, \mathrm{n}^{+}}(t) & 0 & \cdots & 0 \\
\vdots & \vdots & \vdots & \vdots & \ddots & \vdots & \vdots & \vdots & \vdots \\
\mathrm{V}_{\mathrm{n}}^{+} & \mathrm{f}_{\mathrm{n}^{+},+1}(t) & 0 & \mathrm{f}_{\mathrm{n}^{+}, 1^{+}}(t) & \cdots & 0 & 0 & \cdots & -\mathrm{f}_{\mathrm{n}^{+}, \mathrm{n}^{-}}(t) \\
\mathrm{V}_{1}^{-} & 0 & -\mathrm{f}_{-1,1^{-}}(t) & 0 & \cdots & 0 & 0 & \cdots & \mathrm{f}_{1^{-}, \mathrm{n}^{-}}(t) \\
\vdots & \vdots & \vdots & \vdots & \cdots & \vdots & \vdots & \ddots & \vdots \\
\mathrm{V}_{\mathrm{n}}^{-} & 0 & -\mathrm{f}_{\mathrm{n}^{-},-1}(t) & 0 & \cdots & \mathrm{f}_{\mathrm{n}^{-}, \mathrm{n}^{+}}(t) & -\mathrm{f}_{\mathrm{n}^{-}, 1^{-}}(t) & \cdots & 0
\end{array}\right|
$$

\subsection{3. contradiction function matrix $M_{K C}$}

The matrix elements of the contradiction function are

a. at the first row is nodes vector $\mathrm{V}_{+1}, \mathrm{~V}_{-1}, \mathrm{~V}_{1}^{+}, \mathrm{V}_{2}^{+}, \cdots, \mathrm{V}_{\mathrm{n}}^{+}, \mathrm{V}_{1}^{-}, \mathrm{V}_{2}^{-}, \cdots, \mathrm{V}_{\mathrm{n}}^{-}$;

b. at the first column is transposed vector of the first row;

c. the other elements of contradiction function matrix $\mathrm{M}_{\mathrm{KC}}$ are:

If the edge $e_{i j}$ exist, then the matrix element

$$
\mathrm{e}_{\mathrm{ij}}=\mathrm{v}_{\mathrm{ij}}(t) / \mathrm{f}_{\mathrm{ij}}(t)=\mathrm{e}_{\mathrm{ij}}(t) ;
$$

otherwise $\mathrm{e}_{\mathrm{ij}}=0$.

Thus the contradiction function matrix

$$
M_{K C}=\left|\begin{array}{ccccccccc} 
& \mathrm{V}_{+1} & \mathrm{~V}_{-1} & \mathrm{~V}_{1}^{+} & \cdots & \mathrm{V}_{\mathrm{n}}^{+} & \mathrm{V}_{1}^{-} & \cdots & \mathrm{V}_{\mathrm{n}}^{-} \\
\mathrm{V}_{+1} & 0 & 2 / \mathrm{I} & \mathrm{e}_{+1,1^{+}}(t) & \cdots & \mathrm{e}_{+1, \mathrm{n}^{+}}(t) & 0 & \cdots & 0 \\
\mathrm{~V}_{-1} & -2 / \mathrm{I} & 0 & 0 & \cdots & 0 & -\mathrm{e}_{-1,1^{-}}(t) & \cdots & -\mathrm{e}_{-1, \mathrm{n}^{-}}(t) \\
\mathrm{V}_{1}^{+} & -\mathrm{e}_{1^{+},+1}(\mathrm{t}) & 0 & 0 & \cdots & \mathrm{e}_{1^{+}, \mathrm{n}^{+}}(\mathrm{t}) & \mathrm{e}_{1^{+}, 1^{-}}(\mathrm{t}) & \cdots & 0 \\
\vdots & \vdots & \vdots & \vdots & \ddots & \vdots & \vdots & \vdots & \vdots \\
\mathrm{V}_{\mathrm{n}}^{+}-\mathrm{e}_{\mathrm{n}^{+},+1}(t) & 0 & -\mathrm{e}_{\mathrm{n}^{+}, 1^{+}}(t) & \cdots & 0 & 0 & \cdots & \mathrm{e}_{\mathrm{n}^{+}, \mathrm{n}^{-}}(t) \\
\mathrm{V}_{1}^{-} & 0 & \mathrm{e}_{1^{-},-1}(t) & -\mathrm{e}_{1^{-}, 1^{+}}(t) & \cdots & 0 & 0 & \cdots & -\mathrm{e}_{1^{-}, \mathrm{n}^{-}} \\
\vdots & \vdots & \vdots & \vdots & \vdots & \vdots & \vdots & \ddots & \vdots \\
\mathrm{V}_{\mathrm{n}}^{-} & 0 & \mathrm{e}_{\mathrm{n}^{-},-1}(t) & 0 & \cdots & -\mathrm{e}_{\mathrm{n}^{-}, \mathrm{n}^{+}}(t) & \mathrm{e}_{\mathrm{n}^{-}, 1^{-}}(t) & \cdots & 0
\end{array}\right|
$$

2.12.4. Properties of the matrix $M_{K E}, M_{K F}, M_{K C}$

The established expression of Matrix $M_{K E} M_{K F}$ and $M_{K C}$, make the graph $\mathrm{G}_{\mathrm{K}}$ is compute-able in machine. Now some properties about these matrices is explained as below:

a the main diagonal element of these three matrices except first row and first column all are zero;

b because of the graph $G_{K}$ is a directed graph, then these three matrices except their first row and first column are negative symmetrical matrix based on the main diagonal line as the axis of symmetry;

c The elements of the matrices is negative i.e. its direction of edge is wrong in supposed supposition; 


\subsection{The Logic Variable $A_{i}^{ \pm n}(x, y, z ; t)$ Acted on Euclidean Space}

One kind of game is involved the three-dimension Euclidean space, such as football, basketball and tennis,etc., even wars by human beings. This kind of game is acting on the Euclidean space, its true value function is not only depended on time $\mathrm{t}$, but also depended on the position in Euclidean space. Denote the three-dimensions of Euclidean space by $x$, $y, z$, then the logic variable is denoted by $A_{i}^{ \pm n}(x, y, z ; t)$, the true-value-function is denoted by $T\left(A_{i}^{ \pm n}(x, y, z ; t)\right)$.

\subsection{The Logic Variable $A_{i}^{ \pm n}(w ; t)$ Acting on Topological Network Space}

Another kind of game is involved topological network space w, such as railway, highway, river channel, etc. Follow 2.13 , denote the logic variable of this kind game by $A_{i}^{ \pm n}(w ; t)$, and the true value function of the logic variable by $T\left(A_{i}^{ \pm n}(w ; t)\right)$.

And the third kind of game is involved topological network space only by relative time step number s not by absolute time $\mathrm{t}$, such as games on chessboard systems. Follow 2.13 . denote the logic variable by $A_{i}^{ \pm n}(w ; s)$ and denote the true value function by $T\left(A_{i}^{ \pm n}(w ; s)\right)$.

2.15 Discrete variable system for the logic variable $A_{i}^{ \pm n}(t)$, logic variable acting on Euclidean space $A_{i}^{ \pm n}(x, y, z ; t)$, and logic variable acting on topological networks space $A_{i}^{ \pm n}(w ; t)$

To the partial information of the sampled-data for the logic variable $A_{i}^{ \pm n}(t)$, logic variable acted on Euclidean space $A_{i}^{ \pm n}(x, y, z ; t)$, and logic variable acted on topological network space $A_{i}^{ \pm n}(w ; t)$, there will be produced three kind of discrete systems, denote them separately by $A_{i}^{ \pm n}(\Delta t), A_{i}^{ \pm n}(x, y, z ; \Delta t), A_{i}^{ \pm n}(w ; \Delta t)$, in them, the $\Delta t$ denote the sample time interval.

Remark3: The detail about the three kinds of games and the discrete time variable system will be explained in succession papers by author.

2.16 Heredity and Variation Theorem

2.16.1. For $t \in\left[t_{0}, t_{1}\right]$, if the 1 -order derivative of contradiction-function $Q_{i j}(t)$ exist and if

$$
\frac{d Q_{i j}(t)}{d t} \neq 0
$$

then $Q_{i j}(t)$ is called variation, or not is called heredity.

2.16.2. Every contradiction-function $\mathrm{R}_{\mathrm{ij}}(t)$ always can be seen an algebra-sum of a constant $\mathrm{C}$ and function $\mathrm{R}_{\mathrm{ij}}(t)$ i.e.

$$
\mathrm{R}_{\mathrm{ij}}(t)=Q_{i j}(t)+\mathrm{C}
$$

and if 


$$
\frac{d Q_{i j}(t)}{d t} \neq 0
$$

then in formulation (5), $Q_{i j}(t)$ is variation and $\mathrm{C}$ is heredity.

2.16.3. Heredity and Variation Theorem

Heredity and variation is a basic law in objective-logic, so that is a basic law, of cause as a mirror image, also in subjective-logic.

Proof: combining the 2.16.1. and 2.16.2. shown as above which can be proved obviously.

\subsection{The Critical-point Theorem}

For the contradiction-function $\mathrm{R}_{\mathrm{ij}}(t)$, if its m-order derivative exist and if these points make

$$
\frac{d^{k} \mathrm{R}_{\mathrm{ij}}(t)}{d t^{k}}=0 \text {,or } \infty, k=0,1,2, \cdots m
$$

Then these points and some special-selected points are called critical-point.

\subsubsection{Property-function Critical-point theorem}

The existence of critical-points will make what some new property-function is born or some old property-function is dead.

Proof: obviously.

Proof is over.

\subsection{Isomorphic-equality $\leftrightarrow$}

If the rank-number of logic variable, $A_{i}^{ \pm s}(t) A_{j}^{ \pm r}(t)$ is equal, i.e. $s=r$, then $A_{i}^{ \pm s}(t)$ and $A_{j}^{ \pm r}(t)$ is called isomorphic-equality, denote isomorphic-equality as

$$
A_{i}^{ \pm s}(t) \leftrightarrow A_{j}^{ \pm r}(t)
$$

Isomorphic-equality $\leftrightarrow$ is satisfied by as below

reflexive law $A_{j}^{ \pm r}(t) \leftrightarrow A_{j}^{ \pm r}(t), A_{i}^{ \pm s}(t) \leftrightarrow A_{i}^{ \pm s}(t)$

symmetrical law if $A_{i}^{ \pm s}(t) \leftrightarrow A_{j}^{ \pm r}(t)$, then $A_{j}^{ \pm r}(t) \leftrightarrow A_{i}^{ \pm s}(t)$

transitive law if $A_{i}^{ \pm s}(t) \leftrightarrow A_{j}^{ \pm r}(t)$, and $A_{j}^{ \pm r}(t) \leftrightarrow A_{m}^{ \pm k}(t)$, then $A_{i}^{ \pm s}(t) \leftrightarrow A_{m}^{ \pm k}(t)$

2.18.1. Similarity between logic variable $A_{i}^{ \pm s}(t)$ and $A_{j}^{ \pm r}(t)$ thinking analogy method

2.18.1.1. Definition: the two logic variable $A_{i}^{ \pm s}(t)$ and $A_{j}^{ \pm r}(t)$ is called similarity, if

a. $A_{i}^{ \pm s}(t) \leftrightarrow A_{j}^{ \pm r}(t)$

b. in the graph $\mathrm{G}_{\mathrm{K}}^{\mathrm{i}}$, and graph $\mathrm{G}_{\mathrm{K}}^{\mathrm{j}}$, their contradiction-function of corresponding edge is proportional i.e.

$\mathrm{R}_{\mathrm{ij}}^{\mathrm{i}}(t)=C \mathrm{R}_{\mathrm{ij}}^{\mathrm{j}}(t), \quad \mathrm{R}_{\mathrm{ij}}^{\mathrm{i}}(t) \in \mathrm{G}_{\mathrm{ij}}^{\mathrm{i}}, \mathrm{R}_{\mathrm{ij}}^{\mathrm{j}}(t) \in \mathrm{G}_{\mathrm{ij}}^{\mathrm{j}}$ in formulation as above $C$ is a constant.

2.18.1.2. If $A_{i}^{ \pm s}(t)$ and $A_{j}^{ \pm r}(t)$ is called similarity, what is denoted as $A_{i}^{ \pm s}(t) \approx A_{j}^{ \pm r}(t)$. The relation $\approx$ is 
satisfied by reflexive law, symmetrical law and transitive law as same as isomorphic-equality $\leftrightarrow$ shown above.

2.18.1.3. Thinking Analogy-able Theorem

Two of logic variable $A_{i}^{ \pm s}(t)$ and $A_{j}^{ \pm r}(t)$ are analogy-able in thinking, if and only if

$$
A_{i}^{ \pm s}(t) \approx A_{j}^{ \pm r}(t)
$$

Proof: based on the definition of similarity, if $A_{i}^{ \pm s}(t)=C A_{j}^{ \pm r}(t)$

Then

$$
A_{i}^{ \pm s}(t) \approx A_{j}^{ \pm r}(t)
$$

In formulation as above, $C$ is a constant, thus they are analogy-able in thinking.

Proof is over.

\section{Conclusion}

As shown above, author has established an axiom system for dialectical logic K-model depend on several laws, some definitions, graph GK and Mozi's principle, and has proved some theorems for dialectical logic K-model. Author also has established three giving-value matrices to logic variable. In fact, author has established a compute-able mathematical model of contradiction by means of Hegel's contradiction opinion for machine. The model would supply a useful manner to THINKING and REASONNING in artificial intelligence. At same time author also has described simply the logic variable involved three-dimension Euclidean space, topology networks space and discrete logic variable system. The advanced properties and details of these as shown as 2.13.,2.14.,2.15.for dialectical logic K-model will be explained in succession papers by the author.

\section{References}

Bencivenga, E. (2000). Hegel's Dialectical Logic, Oxford University, Press.

Kosok, M. (1966). The Formalization of Hegel's Dialectical logic, International Philosophical Quarterly, IPQ6(4): 596-631, January. https://doi.org/10.5840/jpq 19666456

Johnston, Ian (2010). The Mozi: A complete Translation,Hongkong:The Chinese University Press,

Berge, C. (1973). Graph and hyper graphs[M]_Amsterdam:North-Holland Publishing Company,

\section{Copyrights}

Copyright for this article is retained by the author(s), with first publication rights granted to the journal.

This is an open-access article distributed under the terms and conditions of the Creative Commons Attribution license (http://creativecommons.org/licenses/by/4.0/). 